\title{
Comparing the Efficiency of Public Transportation Subunits Using Data Envelopment Analysis
}

Darold T. Barnum, University of Illinois at Chicago

Sue McNeil, University of Delaware

Jonathon Hart, Wilbur Smith Associates

\begin{abstract}
This article discusses the need for a performance measure that compares the efficiencies of subunits within a transportation organization, reflects the diversity of inputs and outputs, and is objective and consistent. The study presents a method for developing such a performance indicator, and illustrates its use with an application to the park-and-ride lots of the Chicago Transit Authority. The proposed method applies Data Envelopment Analysis supplemented by Stochastic Frontier Analysis to estimate efficiency scores for each subunit. The research demonstrates how the scores can provide objective and valid indicators of each subunit's efficiency, while accounting for key goals and values of internal and external stakeholders. The scores can be practically applied by a transit agency to identify subunit inefficiencies, and, as demonstrated by several brief case studies, this information can be used as the basis for changes that will improve both subunit and system performance.
\end{abstract}

\section{Introduction}

Due to shortages of public funds and expanding societal needs, maintaining and improving the performance of public transportation systems are critical for future operations (Kittelson et al. 2003; Sulek and Lind 2000). If public transportation 
is not as efficient as it could be, it provides less service than desirable or requires taxpayers and riders to pay more than necessary.

Improving the efficiency of a transit system's subunits is one way to increase overall efficiency. Of course, maximizing subunit efficiency does not necessarily maximize system efficiency. However, overall system efficiency can be increased by correctly identifying subunit inefficiencies, and then improving subunit performance with changes that are consistent with system structures, goals, and constraints. For example, the efficiency of subcontracted service providers could be compared, as could the efficiency of individual bus routes, different rail lines, park-and-ride lots, rail stations, garages, and paratransit operations. If some of the subunits performing a given type of activity are identified as relatively inefficient compared with others performing the same activity, then management can take action to improve the least efficient ones, thereby improving overall system performance. The challenge lies in identifying and quantifying objective measures that reflect the multiple outputs and inputs common in public transportation.

This article describes a procedure for comparing subunit efficiency, and demonstrates its application to the Chicago Transit Authority (CTA) park-and-ride lots. Park-and-ride facilities are a strategic component of urban mass transportation systems, effectively extending the service area and attracting riders who may not have otherwise used transit. However, with the high cost of construction, land acquisition, and maintenance of parking facilities, a misplaced or underutilitized lot can quickly consume significant resources with little promise for return on investment. The CTA has 17 park-and-ride lots, which not only provide parking for heavy rail passengers but also generate more than $\$ 1$ million annually in net operating income. Similarly, the Chicago commuter rail lines (operated by Metra) provide 68 lots, and Metra has been significantly increasing its lots' capacities to attract more riders (Ferguson 2000). Indeed, park-and-ride facilities have become an integral part of almost all medium and large urban transportation systems in North America (Turnbull et al. 2004).

This discussion addresses problems in developing valid efficiency measures that (1) simultaneously incorporate all resource inputs and all desired outputs, (2) adjust for the influences of environmental variables, (3) are objective and can be rationally interpreted, and (4) provide consistent measurements with which to compare subunit performance. We describe an innovative solution to the problems-Data Envelopment Analysis (DEA). We identify the DEA model used, and the inputs and outputs that are most valued by the CTA. Using these inputs and 
outputs for the 16 lots for which we have data, we apply DEA. We use Stochastic Frontier Analysis to adjust the lots' DEA efficiency scores for environmental factors. Finally, we present several brief case studies of lots that have been identified as inefficient by DEA.

\section{Problems with Measuring Efficiency of Subunits within a Transit Organization}

In economics, efficiency (or more specifically, technical efficiency) is measured by the ratio of output to input (Cooper, Seiford, and Zhu 2004; Färe, Grosskopf, and Lovell 1994). In public transportation, multiple outputs are produced by multiple inputs, so different efficiency rankings may occur depending on the specific output/input ratios chosen for analysis.

For example, although the primary goal of CTA management is to provide parking for heavy rail passengers, the profit derived from these lots also is an important output according to the agency. Thus, the key outputs are (1) number of parked cars, as a proxy for number of passenger trips and (2) parking revenues. Key inputs are (1) number of parking spaces and (2) operating expenses. Of course, other outputs and inputs could be added, but these are key to the CTA and are used to illustrate the process.

Four different efficiency indicators can be constructed from these two outputs and two inputs: cars/expenses, revenue/expenses, revenue/capacity, and cars/ capacity. Table 1 shows the actual performance of four CTA lots on these four ratios.

Table 1. Comparative Efficiency of Four CTA Park-and-Ride Lots, First Quarter 2005

\begin{tabular}{lcccc} 
Lot & $\begin{array}{c}\text { Cars/ } \\
\text { Expenses }\end{array}$ & $\begin{array}{c}\text { Revenue/ } \\
\text { Expenses }\end{array}$ & $\begin{array}{c}\text { Revenue/ } \\
\text { Capacity }\end{array}$ & $\begin{array}{c}\text { Cars/ } \\
\text { Capacity }\end{array}$ \\
\hline A & 3.05 & 4.25 & 1.18 & 0.85 \\
B & 2.88 & 5.70 & 1.51 & 0.77 \\
C & 0.75 & 1.80 & 2.18 & 0.90 \\
D & 1.08 & 2.14 & 2.13 & 1.08
\end{tabular}


Note that Lot $A$ is the best performer on the first measure, $B$ on the second, $C$ on the third, and $D$ on the fourth. Also, $A$ is the worst performer on the third indicator; $B$ is the worst on the fourth; $C$ is the worst on the first; and $D$ is the next to worst on the second. In short, for this actual data, there are no consistently good or consistently bad performers. Further, which indicators would be considered most important would likely depend on one's function at a transit agency. Land developers would probably prefer to use the third indicator, while those most interested in cash flow would prefer the second. Those concerned in cost control would value the first most heavily, and those trying to maximize transit passenger trips would argue for the fourth. Moreover, at least in Chicago, interest groups for each lot certainly would be involved if the efficiency measures were to be used to make decisions about their lot's fate. It is not hard to imagine which ratios the supporters of each lot would argue were key and which were biased.

Worse, this is a very simple situation: there are only 4 efficiency measures and only 4 lots. Most transit experts could easily identify 4 significant inputs and 4 significant outputs, thereby increasing the potential efficiency measures from 4 to 16 . If all CTA lots were compared with all 16 measures, it would make winners and losers even more difficult to identify.

As this example illustrates, even in very simple situations, it often is difficult to compare the overall efficiency levels of the subunits performing a given type of activity. What is needed is a single, comprehensive measure of performance that would objectively identify the poorest performers overall, and then use other techniques to determine the reasons for poor performance and to decide on appropriate actions.

To obtain a comprehensive efficiency measure for comparing the lots, we could aggregate each lot's outputs and inputs with some weighting scheme, and then divide the aggregated outputs by the aggregated inputs. That is, for each lot, we could calculate the following indicator:

Efficiency $=\frac{\text { OutputWeight } t_{1} \times \text { Output }_{1}+\text { OutputWeight }_{2} \times \text { Output }_{2}}{\text { InputWeight }_{1} \times \text { Input }_{1}+\text { InputWeight }_{2} \times \text { Input }_{2}}$

For example, suppose we are most interested in low costs and high revenues. For the outputs we decide to weight revenues at 0.8 and parked cars at 0.2 . For the inputs, we choose to weight costs at 0.7 and capacity at 0.3 . We use these weights 
to calculate the efficiency of each lot. For instance, for Lot $A$, for the first quarter of 2005 its daily revenue (Output $t_{1}$ ) was $\$ 519$, its daily operating expenses (Input $_{1}$ ) were $\$ 122$, its average number of parked cars (Output ${ }_{2}$ ) was 371 , and its capacity (Input $_{2}$ ) was 441 spaces:

$$
\text { Efficiency }(\text { Lot } A)=\frac{0.8 \times 519+0.2 \times 371}{0.7 \times 122+0.3 \times 441}=2.25
$$

We perform calculations using the same weights for the inputs and outputs of each lot, and then compare the values. However, it would be difficult to defend definitively the preceding weighting scheme as being optimal, and it would be equally hard to justify assigning equal weights to each input and output (or to each of the four ratios presented earlier). An objective and consistent procedure for assigning weights is necessary to solve the problem.

\section{Data Envelopment Analysis}

Data Envelopment Analysis (DEA) offers an innovative approach to the problem of objectively assigning weights to compare the efficiency of the subunits of a transportation organization validly. Since the first papers applying DEA to public transportation were published in 1992, the procedure has become increasingly popular for comparing transit organizations with each other (Brons et al. 2005; De Borger, Kerstens, and Costa 2002). However, DEA has not been used to compare subunits within a given transit organization. Herein, we demonstrate the use of DEA for comparing a set of subunits that each performs the same activity within their parent transportation agency. Similar analyses have been conducted to compare the performance of organizational subunits such as bank branches and retail outlets (Cooper, Seiford, and Zhu 2004; Färe, Grosskopf, and Lovell 1994).

DEA uses linear programming to weight and aggregate outputs divided by inputs in a way that results in a single comprehensive efficiency measure, with efficient units scoring exactly 100 percent. The efficiency of a given subunit (or other unit of analysis), often referred to as a Decision Making Unit (DMU), is expressed as a percentage of the efficiency of its most efficient peers. For the case at hand, each park-and-ride lot is a DMU.

A key feature of DEA is that the weighting for variable aggregation can be different for each DMU. For the target DMU, weights are assigned so it will obtain the high- 
est possible efficiency score when it is compared to the other DMUs, when all have been assigned the particular set of weights that is optimal for the target DMU. That is, the procedure is applied to a particular DMU. Then, the entire process is completed for another target DMU, with new weights being assigned to all DMUs that maximize the efficiency of the new target DMU. This process is completed once for each DMU that is in the set being compared. So, if a particular DMU's score is not 100 percent, this tells us that other DMUs are still more efficient even when the weights are set to maximize the score of the given DMU. Once efficiency levels for each DMU have been identified, they can be analyzed to determine why certain DMUs are more efficient than others.

\section{Inputs, Outputs, and DEA Model}

For this illustration, the inputs are (1) number of parking spaces and (2) mean daily operating costs. The outputs are (1) the mean number of cars parked in the lot during the workday and (2) mean daily revenue. Because the mean revenue per car that the lots receive varies from $\$ 1.39$ to $\$ 3.95$, the two output variables reflect different measures of success. All variables are daily averages for the first quarter of 2005. The DEA model is

$$
\max _{u, v} \quad \theta=\frac{\sum_{m=1}^{M} u_{m} y_{m k^{\prime}}}{\sum_{n=1}^{N} v_{n} x_{n k^{\prime}}}
$$

$$
\begin{array}{cc}
\text { subject to } 1 \geq \frac{\sum_{m=1}^{M} u_{m} y_{m j}}{\sum_{n=1}^{N} v_{n} x_{n j}} & \forall j \\
\sum_{n=1}^{N} v_{n} x_{n k^{\prime}}=1 & \\
u_{m}, v_{n}, y_{m j}, x_{n j}>0 & \forall m, n, j
\end{array}
$$

In our case there are $j \mathrm{DMUs}$; that is, $j$ lots, to be evaluated $(j=1, \ldots, 16)$. Each DMU consumes varying amounts of $n$ different inputs $(n=1,2)$ to produce $m$ different outputs $(m=1,2)$. Thus, for example, $\mathrm{DMU}_{j}$ consumes amount $x_{n j}$ of input 
$n$ and produces $y_{m j}$ of output $m$. For all DMUs, $u_{m}$ is the weight by which each $y_{m j}$ is multiplied, and $v_{n}$ is the weight by which each $x_{n j}$ is multiplied. The DMU that is the target of a given evaluation is designated $\mathrm{DMU}_{k^{\prime}}$, and it is compared to all $j$ of the DMUs including itself. The program (3) maximizes the ratio of weighted outputs to the weighted inputs. The weights $u_{m}$ and $v_{n}$ are the variables, and they are changed until the ratio is maximized for the target DMU when those same weights are applied to all DMUs. The value of the ratio, $\theta$, is the efficiency score of $\mathrm{DMU}_{k^{\prime}}$, where $0 \leq \theta \leq 1$ and a fully efficient $\mathrm{DMU}$ receives a score of 1 . Again, note that it is the weights that are the variables, with the outputs and inputs being the values actually observed for each lot. The DEAs in this study were conducted with the Efficiency Measurement System (EMS) software developed by Scheel (2003), which converts the fractional program in (3) into a linear program before solving.

\section{Unadjusted DEA Efficiency Scores}

The initial efficiency levels of the various lots, unadjusted for any differences in environmental factors, are shown in Table 2. (Frequently, the efficiency score $\theta$ is written as a percentage, so an efficient DMU will have a $\theta=1$ or 100 percent.)

Table 2. Unadjusted DEA Efficiency Scores

\begin{tabular}{cc} 
Lot & $\begin{array}{c}\text { Unadjusted } \\
\text { Efficiency }(\theta \%)\end{array}$ \\
\hline A & 100.00 \\
B & 100.00 \\
C & 100.00 \\
D & 100.00 \\
E & 94.89 \\
F & 91.85 \\
G & 89.19 \\
H & 85.92 \\
I & 76.25 \\
J & 76.25 \\
K & 70.83 \\
L & 62.48 \\
M & 58.51 \\
N & 54.87 \\
O & 43.56 \\
P & 20.91
\end{tabular}


Of the 16 lots, 4 (A, B, C, and D) are efficient with scores of 100 percent; that is, no other lot is more efficient for each of the four lots' optimal mixes of weights. The other lots show varying degrees of inefficiency. Thus, Lot $E$ is 95 percent efficient, and Lot $\mathrm{P}$ is only 21 percent efficient. Again, note that these efficiency levels are relative; the most efficient lots receive efficiency scores of 100 percent. $A$ given inefficient lot's score identifies how much less efficient it is than its efficient peers when all lots use the weighting that maximizes the efficiency of the given lot.

\section{Adjusting Efficiency Scores for Environmental Factors}

Before analyzing each lot to attempt to determine the reasons for its score, it is informative to adjust the scores for key environmental influences. As typically defined in DEA, environmental factors are influences that are not traditional inputs and outputs, and are not under the control of management (Coelli et al. 2005). Hart ( 2005) found that the number of cars using each CTA lot was influenced by the distance of the lot from the nearest freeway and from the central business district (CBD). The closer the lot was to the nearest freeway, the higher the demand, and the further the lot was from the CBD, the higher the demand. Many other environment variables could be important (Hart 2005; Spillar 1997), but only a limited number can actually be used given the sample size, so we use those that Hart found optimal for the Chicago lots.

Different methods have been utilized for adjusting efficiency scores to control for environmental factors (Coelli et al. 2005; Ruggiero 2004). The method currently in favor, often called the two-stage method, involves first computing the efficiency scores using only true inputs and outputs, as we already have done. In the second stage, environmental influences can be controlled for by regressing the initial efficiency scores on the environmental variables, and then adjusting the initial scores by dividing them by the expected scores.

Ordinary Least Squares (OLS) regression, however, is not a valid method to use when the dependent variable is the DEA efficiency score for several reasons. The dependent variable has an upper limit of 100 percent, and therefore is a censored variable. Using OLS regression when the dependent variable is censored results in biases in parameter estimates (Breen 1996). If such censoring were the only concern, then Tobit regression could be used. But, because we also have to deal with biases caused by inefficiency, as discussed later in this section, Tobit regression is not valid either (Kumbhakar and Lovell 2000). 
Another method for dealing with the censoring of DMU efficiency scores is to use superefficiency scores (Andersen and Petersen 1993) as the dependent variable. For inefficient DMUs, efficiency and superefficiency scores are identical. For an efficient DMU, the superefficiency score identifies the proportion by which its current outputs exceed the level necessary to be efficient. Because only four DMUs were found to be efficient in this study, the superefficiency scores will differ from efficiency scores only for these four. The applicable superefficiency scores are lot D (101\%), lot A (102\%), lot B (117\%), and lot C (193\%). Because superefficiency scores are not censored, a statistical model adjusting for censored variables is not necessary if they are used as the dependent variable.

However, OLS regression still is inappropriate (as is Tobit regression) because of the second problem. Just as most of the lots were inefficient in converting inputs to outputs, it is likely that many of the lots will be inefficient in converting environmental factors to outputs. To account for this possibility, it is necessary to use a statistical model that estimates both normal random fluctuations in the error term and downward biases due to inefficiency, which neither OLS nor Tobit regression do. Therefore, we use Stochastic Frontier Analysis, which adjusts for DMU inefficiency in converting environmental conditions into outputs (Coelli et al. 2005; Kumbhakar and Lovell 2000). (Stochastic Frontier Analysis would be inappropriate if efficiency scores were used as the dependent variable instead of superefficiency scores because, as with OLS, a censored dependent variable will bias estimates.)

The dependent variable, therefore, is the superefficiency score for each parking lot. The environmental variables that influence outputs and thereby the efficiency scores are "distance of the parking lot from the central business district" (relationship expected to be positive) and "distance of the parking lot from the nearest freeway" (relationship expected to be negative).

For $\mathrm{DMU}, \mathrm{y}_{j}$ is the superefficiency score; $x_{1 j}$ is the distance of the parking lot from the CBD; $x_{2 j}$ is the distance of the parking lot from the nearest freeway; $v_{j}$ is the two-sided noise component of the error term; and $u_{j}$ is the nonnegative technical inefficiency component of the error term. The noise component $v_{j}$ is assumed to be normally and independently distributed. The inefficiency component $u_{j}$ must be greater than or equal to zero, and is assumed to be exponentially and independently distributed. The model is:

$\ln y_{j}=\beta_{0}+\beta_{1} \ln x_{1 j}+\beta_{2} \ln x_{2 j}+v_{j}-u_{j}$ 
We conducted the analysis with the Frontier Analysis package in Stata 9 (StataCorp 2005). The results are shown in the Table 3. Note that this model is estimated using the maximum likelihood method, not the least squares method, so R-Square statistics are not available.

Table 3. Parameter Estimates

\begin{tabular}{lccc} 
Variable & $\beta$ & Std. Error & $\mathbf{P}>|\mathbf{z}|$ \\
\hline Constant & -0.400 & $4.54 \mathrm{E}-06$ & 0.000 \\
$x_{1}$ (distance from CBD) & 0.195 & $1.65 \mathrm{E}-06$ & 0.000 \\
$x_{2}$ (distance from freeway) & -0.108 & $2.82 \mathrm{E}-07$ & 0.000 \\
\hline Log likelihood = -.102; Wald chi-square $(2)=3.19 \mathrm{e}+11$; Prob $>$ chi-square $=0.000$
\end{tabular}

As expected, the greater the distance of the lot from the CBD $\left(x_{1}\right)$, the higher the efficiency, and the greater the distance of the lot from the nearest freeway $\left(x_{2}\right)$, the lower the efficiency. Thus, it is reasonable to use the expected values predicted by this equation to adjust the uncorrected efficiency scores. The values for adjusted efficiency scores are shown in Table 4.

Table 4. Adjusted Efficiency Scores

\begin{tabular}{cc} 
Lot & $\begin{array}{c}\text { Adjusted } \\
\text { Efficiency }(\theta \%)\end{array}$ \\
\hline B & 100.00 \\
C & 100.00 \\
E & 100.00 \\
H & 97.50 \\
F & 95.52 \\
G & 93.89 \\
D & 86.70 \\
I & 82.10 \\
J & 76.90 \\
L & 66.00 \\
M & 59.50 \\
N & 59.37 \\
K & 51.66 \\
A & 47.75 \\
O & 44.10 \\
P & 22.04
\end{tabular}




\section{Interpreting the Case Study Results}

Identification of lot efficiencies, with both original and adjusted scores, is the first step. Next the "red flag" test should be used to identify lots that should receive further attention (Barnum 1987). Low efficiency scores make it easy to identify those lots that need thorough examination. Obvious examples here are lots $\mathrm{O}$ and $\mathrm{P}$, which scored at the bottom of both sets of efficiency scores; lot $\mathrm{K}$, which had a significant decrease in efficiency between the unadjusted and adjusted efficiency scores; and lot A which was efficient before adjustment for environmental factors but significantly inefficient after taking its favorable environment into account.

In some cases, uncorrectable factors or other justifiable reasons account for the low scores. In other cases, the low scores may identify lots that can and should be improved. To illustrate the method, we next discuss the examinations of lots A and $P$ that resulted from their flagged efficiency scores.

One of the flagged lots was lot A, whose score dropped from 100 percent to 48 percent after being adjusted for environmental factors. Discussions with CTA personnel uncovered the reasons for this decrease. Lot $A$ competes for business with lot $C$ because they are very close to each other. However, both lots are on the same freeway from the suburbs, with lot $C$ being slightly further from the city. That short stretch of freeway is very congested during rush hour. Hence, even though lots A's and $C$ 's environmental factors are almost identical, lot $C$ is likely to get many more cars, thus decreasing the adjusted efficiency for lot $\mathrm{A}$.

Of course, lot $A$ is efficient when only the outputs and CTA resource inputs are considered; that is, the unadjusted efficiency. From the viewpoint of the actual inputs and outputs, the lot is performing well when compared to other lots. Further, it is unlikely that the number of lot A parkers could be increased without adversely affecting lot $C$.

It was suggested that lot A's revenue output could be increased by raising the rate from $\$ 2$ to $\$ 3$ for the first 12 hours. The rate at lot $C$ already is $\$ 3$, so it is likely that if lot A's rate were increased, its revenue would increase much more than the number of parkers decreased. This suggested change may be implemented by the CTA.

Also, lot A's operating expenses are relatively high due to the fact that personnel are on duty to collect parking fees. In lot $C$ and all other lots, parkers deposit their own parking fees. The increase in perceived security provided by parking attendants would attract more parkers to lot $A$, if not for the fact that even more per- 
ceived security is provided at lot $C$. The suburban police patrol lot $C$ and "maintain an almost constant presence" (Hart 2005, p. 92). CTA resources are not used for this police protection, so lot C's operating expenses remain low, thereby giving it a big boost in efficiency. Given the recent increase in concerns for security at transit facilities, perhaps the CTA could obtain similar protection for lot A (which is a multistory structure near the airport). This could decrease the CTA's input costs, thereby increasing the lot's efficiency.

In a somewhat similar situation, lot $\mathrm{P}$ competes with lot $\mathrm{K}$, with not enough parkers to fill both lots. For the unadjusted efficiencies, lot $\mathrm{K}$ is the clear winner. But, lot $\mathrm{K}$ also enjoys very favorable environmental variables while lot $\mathrm{P}$ does not. Thus, it is not surprising that lot K's efficiency decreases from 71 to 52 percent when we adjust for environmental factors, while lot P's efficiency increases slightly from 21 to 22 percent.

As seen in Table 4, lot $\mathrm{P}$ has the lowest efficiency of all lots for both the unadjusted and the adjusted efficiency scores. This is the result of a relatively large lot with average operating expenses, but with very low occupancy and revenues. These outcomes in turn are likely influenced by the nearby presence of lot $K$, which has in the past been considered a more desirable place to park. At the time these data were collected, lots $K$ and $P$ were at terminals at the western ends of two branches of the same rapid transit line, and these two branches merged before the line entered the CBD. However, the branch to lot $\mathrm{K}$ not only was closer to a freeway, but also it provided more hours of service, and the branch to lot $P$ was impeded because of major upgrades to the tracks and terminals. Recently, however, the branches have been separated into two independent lines, and the frequency of service on each has been doubled. The enhanced service and the readily available parking is expected to increase passengers and parkers for both terminals. If demand does not increase in a reasonable time, however, it might be worth considering the impact of downsizing or closing lot $P$, since its current demand could be absorbed by lot $\mathrm{K}$. Of course, there may well be factors other than DEA efficiency scores that make such a suggestion infeasible.

Herein we have given only brief analyses of a few lots as examples of the process. Once a lot is flagged as the result of a low efficiency score, a thorough examination of the lot should be made to determine the reasons for the low score. As demonstrated above, sometimes the factors influencing the scores are correctable, and other times they are not. 
As was done in this case, flagged subunits should be subjected to joint examinations by transit agency personnel and the DEA analysts, and not be examined by just one group or the other. To best identify and correct problems, both a deep understanding of the transit system and an understanding of the analytical method must be integrated. In the present case, such an examination took place as part of the ongoing partnership between the CTA and the Urban Transportation Center at the University of Illinois at Chicago. This joint analysis provided much of the information in this section.

\section{Conclusions}

Data Envelopment Analysis, augmented by Stochastic Frontier Analysis, is a methodology for identifying and comparing the efficiencies of a set of subunits within an organization that recognizes the diversity of inputs and outputs that impact and influence performance, and that provides insights into the differences in performance. Once management has identified the key outputs and inputs of a given type of subunit, then DEA can be applied to identify a comprehensive efficiency score for each subunit of that type. Because this score results in each subunit attaining the maximum reported efficiency when compared to its peers, it is unbiased by particular points of view about the importance of various inputs or outputs, and hence is a much more valid summary measure than typically utilized. This measure can be adjusted to account for differences in environmental factors affecting the subunits, if necessary. Once the original or adjusted measures of efficiency are obtained, then those subunits with low scores can be carefully studied to develop plans of action to improve their efficiencies.

\section{Acknowledgements}

This project was partially supported by the Chicago Transit Authority under contract to the Urban Transportation Center at the University of Illinois at Chicago, which made this research possible. We especially thank Mark Patzloff, Eric Holeman, and Vincent Nwokolo for generously offering much insight into the CTA's park-and-ride operations as well as very quickly providing necessary data. Without the substantial knowledge of these three individuals and their enthusiastic support, the study could not have been completed. Our work with them is an example of the productive partnership between the CTA and UIC, in which inputs of industry acumen by CTA personnel and inputs of research skills by university 
personnel are combined to produce valuable outputs that would not have been attained by either group alone. Of course, in this research, our ability to interpret correctly what we were told may be lacking, so any errors or misstatements in this article are entirely our own. Likewise, all identifications of lot efficiency are ours alone, and have not been endorsed either by the aforementioned individuals or by the CTA.

\section{References}

Andersen, P., and N. C. Petersen. 1993. A procedure for ranking efficient units in data envelopment analysis. Management Science 39 (10): 1261-1265.

Barnum, D. T. 1987. Evaluating transit: the performance tree method. In Managing urban transportation as a business, E. Bers, and C. Hendrickson, eds. New York: American Society of Civil Engineers, 216-230.

Breen, R. 1996. Regression models: censored, sample-selected, or truncated data. Thousand Oaks, CA: Sage Publications.

Brons, M., P. Nijkamp, E. Pels, and P. Rietveld. 2005. Efficiency of urban public transit: a meta analysis. Transportation 32 (1): 1-21.

Coelli, T. J., D. S. P. Rao, C. J. O'Donnell, and G. E. Battese. 2005. An introduction to efficiency and productivity analysis. New York, NY: Springer.

Cooper, W. W., L. M. Seiford, and J. Zhu. 2004. Handbook on data envelopment analysis, Boston, MA: Kluwer Academic Publishers.

De Borger, B., K. Kerstens, and A. Costa. 2002. Public transit performance: what does one learn from frontier studies? Transport Reviews 22 (1): 1-38.

Färe, R., S. Grosskopf, and C. A. K. Lovell. 1994. Production frontiers. Cambridge England: Cambridge University Press.

Ferguson, E. 2000. Parking management and commuter rail: the case of northeastern Illinois. Journal of Public Transportation 3 (2): 99-121.

Hart, J. D. 2005. Strategies for effective design and deployment of park-and-ride facilities. Thesis. Chicago, IL: College of Urban Planning and Public Affairs, University of Illinois at Chicago.

Kittelson Associates, Urbitran Associates, LKC Consulting Services, MORPACE International, Queensland University of Technology, and Nakanishi, Y. 2003. A 
guidebook for developing a transit performance-measurement system. Transit Cooperative Research Program Report 88. Washington, DC: Transportation Research Board.

Kumbhakar, S., and C. A. K. Lovell. 2000. Stochastic frontier analysis, Cambridge England: Cambridge University Press.

Ruggiero, J. 2004. Performance evaluation in education: modeling educational production. In Handbook on data envelopment analysis, W.W. Cooper, L. M. Seifert, and J. Zhu, eds. Boston, MA: Kluwer Academic Publishers, 323-348.

Scheel, H. 2003. EMS: Efficiency Measurement System. http://www.wiso.uni-dortmund.de/lsfg/or/scheel/ems/.

Spillar, R. J. 1997. Park-and-ride planning and design guidelines. New York: Parsons Brinckerhoff.

StataCorp. 2005. Stata Statistical Software. Release 9.

Sulek, J. M., and M. R. Lind. 2000. A systems model for evaluating transit performance. Journal of Public Transportation 3 (1): 29-47.

Turnbull, K. F., R. H. Pratt, J. E. I.Evans, and H. S. Levinson. 2004. Park-and-ride/pool. In Traveler Response to Transportation System Changes Handbook. Washington, DC: Transportation Research Board, 3.i-3.93.

\section{About the Authors}

DAROLD T. BARNUM (dbarnum@uic.edu) is a professor of management and of information and decision sciences at the University of Illinois at Chicago. He formerly was an associate director at the Indiana University Institute for Urban Transportation, where he participated in the training of transit managers from across the nation. His research focuses on performance measurement, and he has published in Management Science, International Transactions in Operational Research, Interfaces, IEEE Transactions on Engineering Management, and Transportation Research Record. He is a member of the TRB Public Transportation Marketing and Fare Policy Committee.

Sue McNeil (mcneil@ce.udel.edu) is a professor of civil and environmental engineering at the University of Delaware. She formerly was director of the University Illinois at Chicago Urban Transportation Center, and professor of urban planning and policy. Her research and teaching interests focus on transportation infra- 
structure management with emphasis on the application of advanced technologies, economic analysis, analytical methods, and computer applications. She has published in the Journal of Transportation Engineering, Journal of Infrastructure Systems, Transportation Research Record, Journal of Public Works Management and Policy, and Journal of Urban Planning and Development. She is an associate editor for the Journal of Infrastructure Systems, chair of the TRB Transportation Asset Management Committee, and a member of the TRB Executive Committee.

JOHNATHON HART (jhart@wilbursmith.com) is a transportation analyst and project manager with Wilbur Smith Associates' Transportation Finance and Technology division. Prior to his current position, Mr. Hart was a graduate student at the University of Illinois at Chicago. While attending UIC, Mr. Hart studied under Professor McNeil, then director of the Urban Transportation Center, where they worked to develop new methodologies in the optimal siting and sizing of parkand-ride lots for the Chicago Transit Authority. 